\title{
A Low Nuclear Recoil Energy Threshold for Dark Matter Search with CRESST-III Detectors
}

\author{
M. Mancuso ${ }^{1}$ - G. Angloher ${ }^{1}$ - P. Bauer ${ }^{1}$ - A. Bento ${ }^{1,8}$ - C. Bucci ${ }^{2}$. \\ L. Canonica ${ }^{2,9}$ - A. D'Addabbo ${ }^{2}$ X. Defay ${ }^{3}$ - A. Erb $^{3,10}$. \\ Franz von Feilitzsch ${ }^{3}$. N. Ferreiro Iachellini ${ }^{1}$ - P. Gorla ${ }^{3}$ - A. Gütlein ${ }^{4,5}$. \\ D. Hauff ${ }^{1}$ - J. Jochum ${ }^{6}$ - M. Kiefer ${ }^{1}$ - H. Kluck ${ }^{4,5}$ - H. Kraus ${ }^{7}$. \\ J. C. Lanfranchi ${ }^{3}$ - A. Langenkämper ${ }^{3}$. J. Loebell ${ }^{6}$ - E. Mondragon ${ }^{3}$. \\ A. Münster ${ }^{3}$ - C. Pagliarone $^{2}$ - F. Petricca ${ }^{1}$ - W. Potzel ${ }^{3}$ - F. Pröbst ${ }^{1}$. \\ R. Puig ${ }^{4,5}$ - F. Reindl ${ }^{4,5}$ - J. Rothe ${ }^{1}$ - K. Schäffner ${ }^{11}$ - J. Schieck ${ }^{4,5}$. \\ V. Schipperges ${ }^{6}$ - S. Schönert ${ }^{3}$ - W. Seidel ${ }^{1}$ - M. Stahlberg ${ }^{4,5}$. \\ L. Stodolsky ${ }^{1}$ - C. Strandhagen ${ }^{6}$ - R. Strauss ${ }^{1}$ - A. Tanzke ${ }^{1}$. \\ H. H. Trinh Thi ${ }^{3}$. C. Türkoglu ${ }^{4,5}$ - M. Uffinger ${ }^{6}$ - A. Ulrich ${ }^{3}$ - I. Usherov ${ }^{6}$. \\ S. Wawoczny ${ }^{3}$ - M. Willers ${ }^{3}$. M. Wüstrich ${ }^{1}$
}

Received: 7 November 2017 / Accepted: 30 April 2018 / Published online: 21 May 2018

(C) The Author(s) 2018

\begin{abstract}
The CRESST-III experiment (Cryogenic Rare Events Search with Superconducting Thermometers), located at the underground facility Laboratori Nazionali del Gran Sasso in Italy, uses scintillating $\mathrm{CaWO}_{4}$ crystals as cryogenic calorimeters to search for direct dark matter interactions in detectors. A large part of the parameter space for spin-independent scattering off nuclei remains untested for dark matter particles with masses below a few $\mathrm{GeV} / \mathrm{c}^{2}$, despite many naturally motivated theo-
\end{abstract}

M. Mancuso

michele.mancuso@mpp.mpg.de

1 Max-Planck-Institut für Physik, 80805 München, Germany

2 INFN Laboratori Nazionali del Gran Sasso, 67010 Assergi, Italy

3 Physik-Department E15, Technische Universität München, 85747 Garching, Germany

4 Institut für Hochenergiephysik der ÖAW, 1050 Wien, Austria

5 Atominstitut, Vienna University of Technology, 1020 Wien, Austria

6 Eberhard-Karls-Universit at Tübingen, 72076 Tübingen, Germany

7 Department of Physics, University of Oxford, Oxford OX1 3RH, UK

8 LIBPhys, Departamento de Fisica, Universidade de Coimbra, 3004516 Coimbra, Portugal

9 Massachusetts Institute of Technology, Cambridge, MA 02139, USA

10 Walther-Meiner-Institut für Tieftemperaturforschung, 85748 Garching, Germany

11 GSSI-Gran Sasso Science Institute, 67100 L'Aquila, Italy 
retical models for light dark matter particles. The CRESST-III detectors are designed to achieve the performance required to probe the low-mass region of the parameter space with a sensitivity never reached before. In this paper, new results on the performance and an overview of the CRESST-III detectors will be presented, emphasizing the results about the low-energy threshold for nuclear recoil of CRESST-III Phase 1 which started collecting data in August 2016.

Keywords Cryogenic detectors · Dark matter - Rare-event searches

\section{Introduction}

The direct search for dark matter (DM) is one of the most exciting fields in astroparticle physics. Many cosmological observations at different scales agree on the existence of DM, ascribing $\sim 25 \%$ of the Universe's composition to it. In this context, a variety of experiments have been performed in order to investigate the different possible DM candidates arising from theory. The search for dark matter particles is currently carried out with three experimental approaches: production at particles collider experiments, indirect detection via the search of its decay products in cosmic rays and, lastly, direct detection of nuclear recoils produced by dark matter elastic scattering off target nuclei.

The CRESST experiment (Cryogenic Rare Event Search with Superconducting Thermometers) belongs to the last group, investigating the scatters off of three different nuclei in $\mathrm{CaWO}_{4}$ crystals: calcium, tungsten and oxygen. Two possible approaches are possible to improve the experimental sensitivity to DM scattering elastically off nuclei: increase the active volume of the detector in order to statistically increase the interactions of DM, and lower the energy threshold to a value where more events are expected. In this context, the design for the CRESST-III detector is optimized to increase the sensitivity to low-energy nuclear recoils and the background rejection capabilities. To fully exploit the developed modules' potential, we attempt to define the optimal energy threshold during analysis with a method based on the optimum filtering technique. Follows a description of the CRESST-III experiment and a detailed explanation of the solution adopted to define the energy threshold.

\subsection{The CRESST Experiment}

Cryogenic experiments currently provide the best sensitivity for light dark matter particles thanks to their low threshold. The CRESST-III experiment, located at the underground facility Laboratori Nazionali del Gran Sasso in Italy, uses scintillating $\mathrm{CaWO}_{4}$ crystals as cryogenic calorimeters to search for direct dark matter interaction in detectors. The CRESST-II result in 2015 [1] is leading the field of direct dark matter search for dark matter masses below $1.7 \mathrm{GeV} / \mathrm{c}^{2}$, extending the reach of direct search to the sub-GeV/c ${ }^{2}$ mass region. In CRESST-III, an array of 10 scintillating $\mathrm{CaWO}_{4}$ crystals of $\sim 25 \mathrm{~g}$ each is read out simultaneously as cryogenic calorimeters and scintillating detectors.

The expected recoil spectrum of dark matter particle-nucleus elastic scattering shows an exponential raise toward low energies, and thus, a small energy threshold will 
enhance the sensitivity of the detector. This is the reason why after the 2015 CRESSTII result the R\&D activity mainly focused on threshold improvement. Geometry and sensor design have been optimized in order to increase the signal amplitude [2].

Due to the low event rate of dark matter particle-nucleus elastic scattering, an extremely low background environment is required. If not suppressed, events caused by radioactive background would overcome the signal expected from dark matter particles. To shield against cosmic radiation, the setup is installed in a deep underground site under the Gran Sasso massif in Italy, covered by at least 1400 meters of rock. Ambient radioactivity originating from the surroundings is also suppressed as much as possible by multiple layers of passive shielding. These are composed of $14 \mathrm{~cm}$ of radiopure copper directly surrounding the experimental volume, followed by $20 \mathrm{~cm}$ of lead with a low ${ }^{210} \mathrm{~Pb}$ activity of $35 \mathrm{~Bq} / \mathrm{kg}$. A neutron moderator of $50 \mathrm{~cm}$ polyethylene is placed outside the $\mathrm{Cu}$ and $\mathrm{Pb}$ shields, and the atmosphere inside the entire shielding is constantly flushed with nitrogen gas in order to prevent radon from penetrating it. With the moderator installed, the remaining neutron flux would be dominated by muon-induced neutrons in the lead of the shielding. This eventuality is prevented by the muon veto system surrounding the entire setup and by a 5 -cm-thick polyethylene layer inside the $\mathrm{Pb} / \mathrm{Cu}$ shields.

\section{The CRESST-III Detector Module}

Each detector consists of a $20 \times 20 \times 10 \mathrm{~mm}^{3} \mathrm{CaWO}_{4}$ scintillating crystal of $\sim 25 \mathrm{~g}$ and a second smaller nearby cryogenic calorimeter made of silicon on sapphire (SOS) for the detection of the scintillating light. This detector concept is thoroughly discussed in [3]. To measure energy deposition, both calorimeters are equipped with transition edge sensors (TES), designed to provide thresholds of $20-100 \mathrm{eV}$ for the $\mathrm{CaWO}_{4}$ crystals and 5-20 eV for the silicon on sapphire detectors (see Fig. 1).

Despite the effort to shield the setup, the unavoidable natural radioactivity from the material used to build it would be enough to shade a DM signal. To prevent this, a double channel readout (direct energy deposition and light emission) allows eventby-event particle identification which is used for suppressing background induced by charged particles [4]. In addition, CRESST-III detectors are also equipped with a fully scintillating housing and instrumented holders to veto a possible background originating from surrounding surfaces. This innovative active holding system allows to suppress induced thermal signals from particle interaction in the holding material see Fig. 2. In particular, looking at the unblinded training data from one detector operated in the present run [5], we observe a total rate in the ROI (between $100 \mathrm{eV}$ and $40 \mathrm{keV}$ ), without making use of any veto of $(8.6 \pm 0.8) \times 10^{-3} \frac{\mathrm{cts}}{\mathrm{keV} \mathrm{kg} \mathrm{day}}$. Accounting for the aforementioned instrumented holder, we can reject events originated in the holding materials leading to a background rate of $(7.9 \pm 0.8) \times 10^{-3} \frac{\mathrm{cts}}{\mathrm{keV} \mathrm{kg} \mathrm{day}}$. At last we apply event-by-events particle identification to discriminate $\gamma / \beta$ and $\alpha$ particles to further reduce the rate down to $(0.65 \pm 0.26) \times 10^{-3} \frac{\mathrm{cts}}{\mathrm{keV} \mathrm{kg} \mathrm{day}}$. Considering all veto systems, the surviving events are all below $3 \mathrm{keV}$ where the light yield discrimination power is reduced. The reported rates are computed taking into account all the cut efficiencies as report in [5]. 

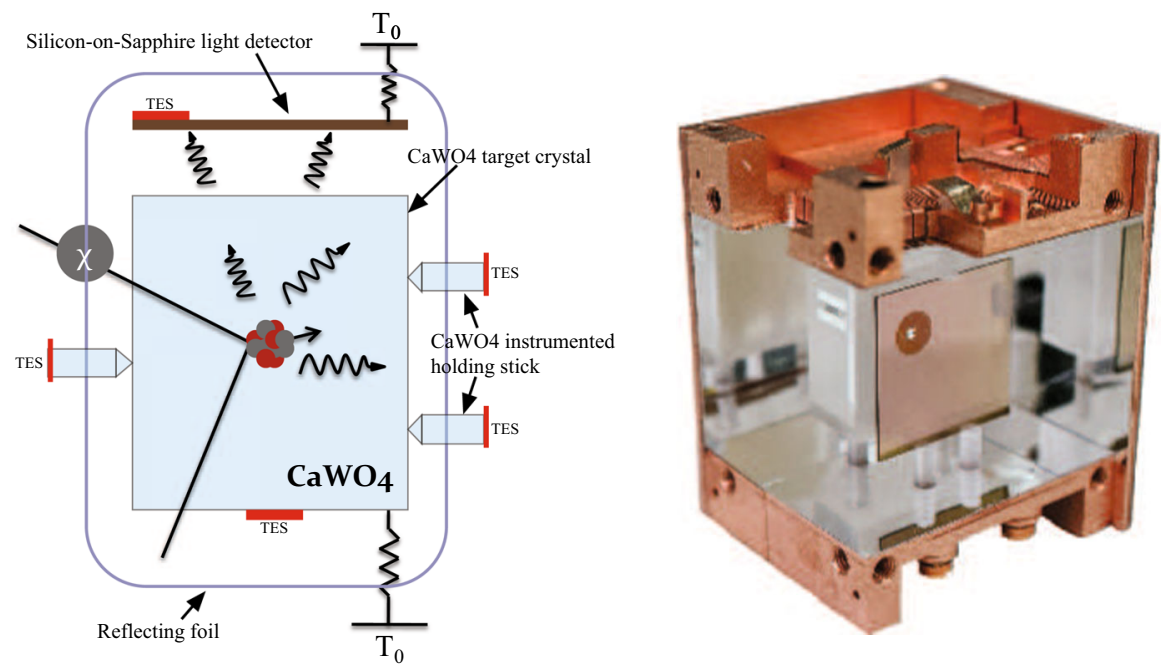

Fig. 1 Left: schematic view of the detector design for CRESST-III modules. A DM particle scattering off nucleus produces phonons, measured by the TES deposited on the target crystal, and photons, collected by the SOS light detector. Both detectors are weakly coupled to a thermal bath at $T_{0} \sim 15 \mathrm{mK}$. Right: picture of a CRESST-III detector module (Color figure online)

\section{Threshold}

The excellent sensitivity and, consequently, the low-energy thresholds of these detectors are their most appealing features, giving the possibility to probe DM particles with masses below $\sim 1 \mathrm{GeV} / \mathrm{c}^{2}$ with an unprecedented sensitivity.

An energy threshold optimization starts with the detector's design, followed by noise optimization in the setup and ends with the analysis. The latter consists essentially of noise filtering, since the threshold is chosen as a function of the noise level to avoid tagging noise fluctuations as physical events. For this purpose, we decided to record the entire data and run a software trigger after filtering the noise. A dedicated matchedfilter which maximizes signal-to-noise ratio after filtering is used. The transfer function $H(j \omega)$ we implemented [6] is built using only the noise power spectrum $\mathcal{N}(\omega)$ and the response function $s(t)$ of the detector after a particle interaction.

$$
H(j \omega)=K^{\prime} \frac{S^{*}(\omega)}{\mathcal{N}(\omega)} \mathrm{e}^{-j \omega \tau_{M}} .
$$

Since the signal shows an improvement in the signal-to-noise ratio when filtered, the threshold can be reduced compared to raw data. This effect can be appreciated in Fig. 3.

At low energy and especially at threshold energy, noise has about the same amplitude of the signal. In this condition, pulse shape analysis fails to distinguish between the two. In this regime, random fluctuations in the noise become an irreducible background contribution, possibly misleading the interpretation of the results near threshold (where the DM signal is expected) if not accounted for. 

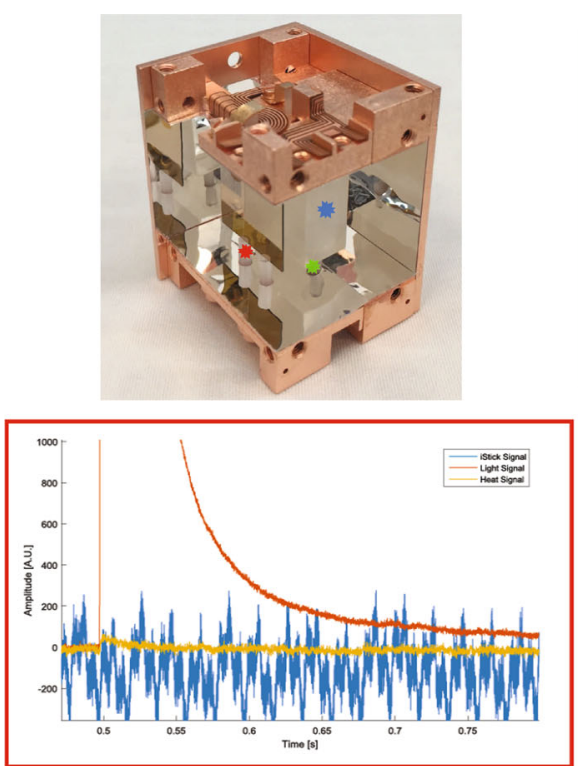
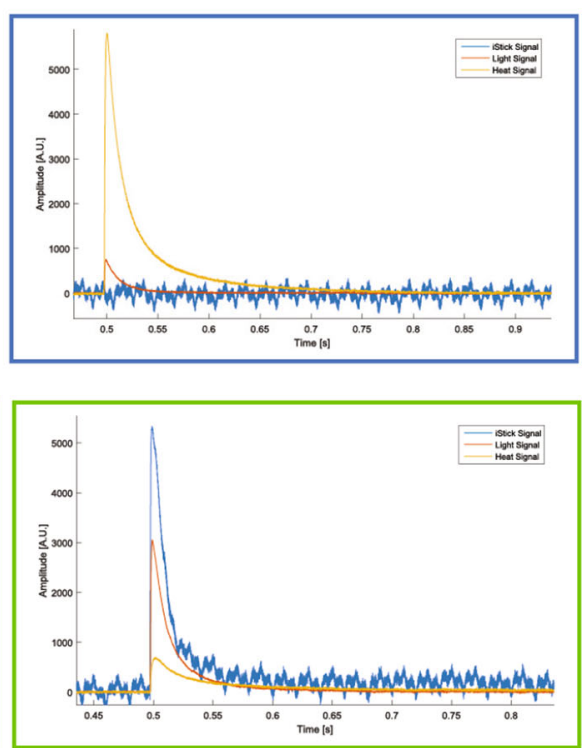

Fig. 2 A picture of a CRESST-III detector module. Top-left: In the picture, we see three possible interaction points corresponding to the recorded pulses displayed in the colored panels. Each trace in the panels describes the record of 1 channel during the particle interaction: Yellow is the main absorber signal, red is the light detector signal, and blue is the holding veto signal (iStick). From the ratio of the amplitude of the coincidence pulse, it is possible to locate the interaction point. Top-right (blue) panel: when an event occurs in the main absorber, where all the energy is deposited, a fraction of which is converted in photons which, in turn, are collected and measured by the light detector. The veto channel is silent. Bottom-left (red) panel: this event occurs in the light detector holding material, the light detector signal is gigantic in respect to the main absorber signal and thus could be rejected as it has a wrong light yield. Bottom-right (green) panel: the interaction takes place in the absorbers' holding material; the veto channel receives a signal so the event can be rejected. In case the veto had not been present, the small induced signal in the main absorber could be misidentified as a small energy deposit (Color figure online)
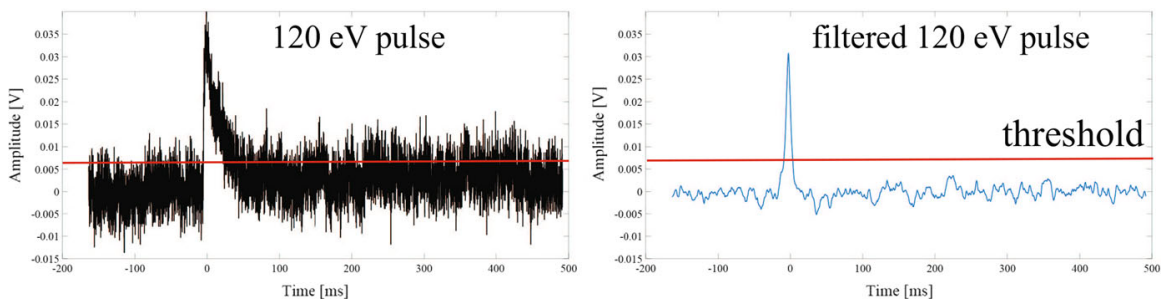

Fig. 3 120-eV Pulse recorded with one CRESST-III detector. On the left the raw pulse is shown, and on the right panel the same pulse after the filter is shown. The red line represents the threshold value achievable after filtering (Color figure online)

A common choice to define the trigger threshold value is 5 times the sigma value of the baseline noise distribution. We propose, instead, to set it according to the total rate of background contribution due to triggering in the noise. 
Given the filter used, the residual noise distribution can be described by a Gaussian function. In this case, the probability $P_{d}\left(x_{\max }\right)$ that the maximum value of the samples exceeds the threshold value in a time window of length $d$ containing only noise is [7]:

$$
P_{d}\left(x_{\max }\right)=\frac{d}{\sqrt{2 \cdot \pi} \cdot \sigma} \cdot\left(\mathrm{e}^{-\left(\frac{x_{\max }}{\sqrt{2} \sigma}\right)^{2}}\right) \cdot\left(\frac{1}{2}+\frac{\operatorname{erf}\left(x_{\max } /(\sqrt{2} \sigma)\right)}{2}\right)^{d-1}
$$

where $d$ is the number of samples in a trigger window, $\sigma$ is the standard deviation of the noise distribution, and $\operatorname{erf}(x, \sigma)$ is the Gaussian error function.

In Fig. 4, the black distribution describes the reconstructed energy of the noise triggers in detector $\mathrm{A}^{1}$ as a function of the energy threshold. In order to be compared with other background contributions, this distribution is normalized to counts/(kg day). In Fig. 4, the blue line is the result of the fit with Eq. 2. In the fit, we left the parameters $d$ and $\sigma$ free and obtained a value which is statistically compatible with the input values for every detector considered in this analysis.

Among the 10 detectors deployed in CRESST-III phase 1, five were studied with this analysis method, which is thoroughly discussed in [7]. We decided to set the energy threshold for triggering based on a rate of counts in the noise that would be acceptable in light of the total background rate. To do so, once the parameters $d$ and $\sigma$ are obtained by fitting the maxima distribution, Eq. 2 can be integrated from the threshold value to infinity in order to obtain the total rate of counts in the noise above threshold. This background must make a negligible contribution to the energy spectrum and thus chose a threshold value in agreement with this requirement.

In CRESST-III, the background at low-energy region is still under investigation, that is why the final decision on the acceptable noise trigger rate has not been reached yet. In order to obtain a preliminary results, we consider as acceptable noise trigger rate an interval of 1-100 counts/(kg day) for which the resulting threshold values are reported in Fig. 5. The investigated detectors have thresholds which match the design goal of $100 \mathrm{eV}$ or below. In particular, detector A has a remarkable energy threshold between 22.6 and $26.1 \mathrm{eV}$.

To confirm the validity of this approach, we can investigate the trigger efficiency curve injecting in the data a known number of pulses with different energies close to threshold. For a given threshold value, a trigger efficiency curve represents the percentage of triggered pulses as function of pulses energy (Fig. 4). It can be described with an error function, which has to demonstrate the same $\sigma$ obtained by Eq. 2 and also be equal to the $\sigma$ of the noise distribution. The threshold efficiency must also equal 0 for small pulses well below threshold; otherwise, it would be too low. For detector $\mathrm{A}$, the baseline fluctuation has a $\sigma_{\text {baseline }}=1.01 \pm 0.03$, the maximum distribution has a $\sigma_{\text {max-dist }}=1.07 \pm 0.06$ and lastly the trigger efficiency for $22.6 \mathrm{eV}$ has a $\sigma_{\text {th-eff }}=0.98 \pm 0.03$ all of which are statistically compatible.

\footnotetext{
1 The 10 detector modules of CRESST-III are named from A to J
} 


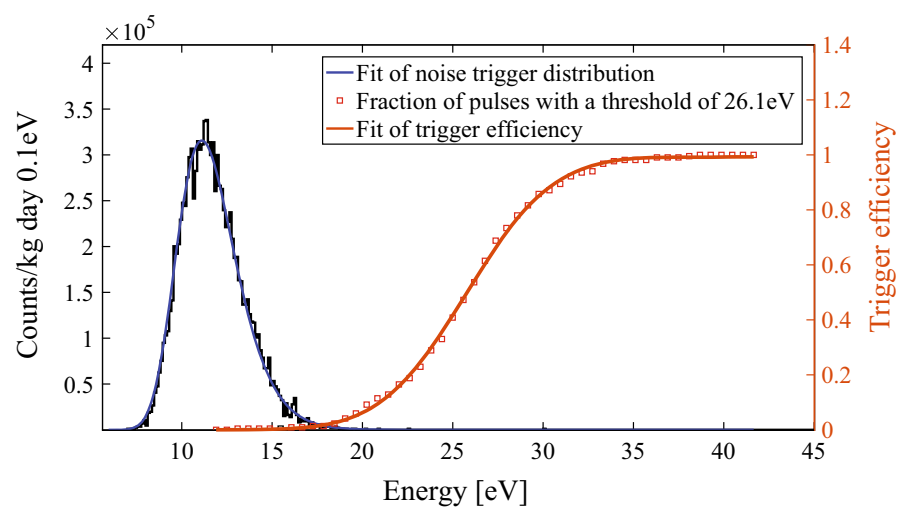

Fig. 4 Results obtained with detector A. Black distribution of maxima in filtered windows containing only noise. Blue fit of the maximum distribution with Eq. 2. Red trigger efficiency curve obtained with a trigger threshold of $26.1 \mathrm{eV}$ (Color figure online)

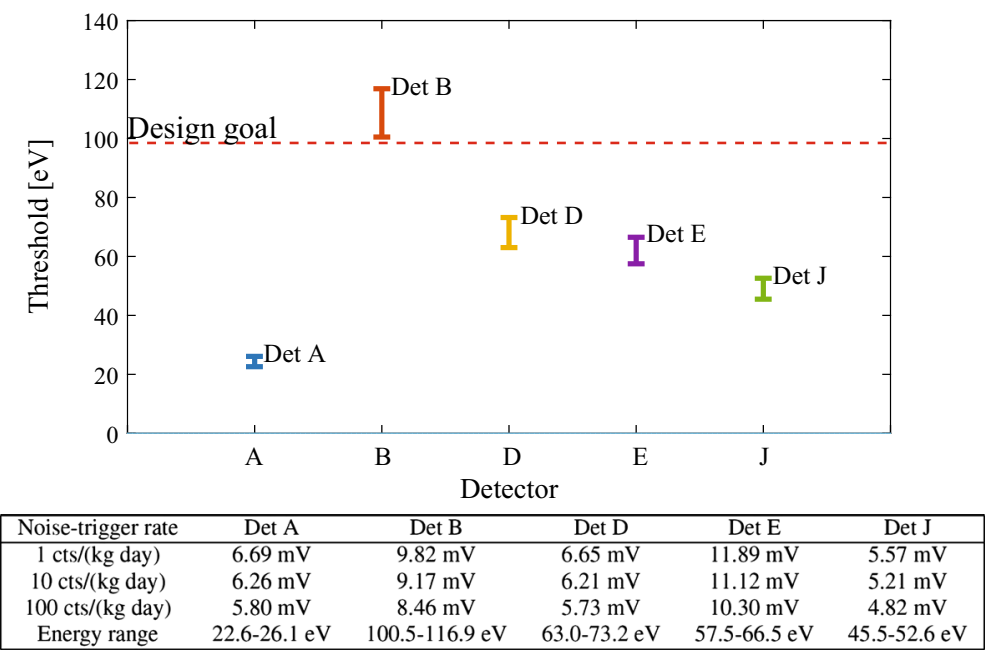

Fig. 5 Summary results of the CRESST-III detector threshold as function of the acceptable trigger rate. Top: visualization of the energy interval for noise trigger rate between 1 and 100 counts/(kg day). Top: table containing the corresponding threshold in $\mathrm{mV}$ of the 3 noise trigger rates considered in this work (Color figure online)

\section{Conclusion}

The CRESST-III detectors use the double readout of heat and light for an event-byevent particle identification strongly reducing the background contributions from the irreducible environmental radioactivity. In order to further reduce the background induced by interaction in the surrounding materials, the CRESST-III detector holders are instrumented giving veto signals for such cases.

We have evaluated the irreducible background contribution caused by trigger in baseline fluctuations in our detectors and described the spectral shape of this noise- 
induced background with an analytical description Eq. 2, which can now also be included in future background models for a better data description. We believe this is an important step forward in rigorous data analysis at threshold energy.

We decided to define the acceptable rate of noise triggers in a range equal to 1100 counts/(kg day) resulted in a sub-100 eV threshold for four CRESST-III detectors, the best achieved so far by massive calorimetric detectors.

Acknowledgements Open access funding provided by Max Planck Society.

Open Access This article is distributed under the terms of the Creative Commons Attribution 4.0 International License (http://creativecommons.org/licenses/by/4.0/), which permits unrestricted use, distribution, and reproduction in any medium, provided you give appropriate credit to the original author(s) and the source, provide a link to the Creative Commons license, and indicate if changes were made.

\section{References}

1. G. Angloher et al., (CRESST) 2016 Eur. Phys. C. C76, 25 (2016). (Preprint 1509.01515)

2. G. Angloher et al., (CRESST) (2015). (Preprint 1503.08065)

3. R. Strauss et al., (CRESST) 2015 Eur. Phys. J. C 75, 352 (2015). (Preprint 1410.1753)

4. R. Strauss et al., Eur. Phys. J. C 74, 2957 (2014). (Preprint 1401.3332)

5. F. Petricca et al., (CRESST) 2017 in 15th International Conference on Topics in Astroparticle and Underground Physics (TAUP 2017) Sudbury, Ontario, Canada, July 24-28, 2017 (2017). (Preprint 1711.07692)

6. E. Gatti, P.F. Manfredi, Processing the signals from solid state detectors in elementary particle physics. Riv. Nuovo Cimento 9(1), 1-146 (1986)

7. M. Mancuso et al., (2017). (Preprint 1711.11459) 